\title{
Global Dynamics of a Harmonically Excited Oscillator with a Play: Numerical studies
}

\author{
Antonio S.E. Chonga,b, Yuan Yue ${ }^{\mathrm{a}, \mathrm{c}}$, Ekaterina Pavlovskaia $^{\mathrm{a}}$, Marian Wiercigroch ${ }^{\mathrm{a}}$ \\ ${ }^{a}$ Centre for Applied Dynamics Research, School of Engineering, University of Aberdeen, Aberdeen AB24 3UE, UK \\ ${ }^{b}$ Facultad de Ciencias Naturales y Matemáticas, Escuela Superior Politécnica del Litoral, P.O. Box 09-01-5863, \\ Guayaquil, Ecuador \\ ${ }^{c}$ Applied Mechanics and Structure Safety Key Laboratory of Sichuan Province, School of Mechanics and Engineering, \\ Southwest Jiaotong University, Chengdu, 610031, China
}

\begin{abstract}
In this paper a harmonically excited linear oscillator with a play is investigated. Direct numerical simulation and numerical continuation techniques were employed to study the system behaviour. To conduct the numerical analysis, the system differential equations were transformed into the autonomous form and were then solved using our newly developed in-house Matlab-based computational suite ABESPOL [1]. The results are presented in form of trajectories and Poincaré maps on the phase plane, bifurcation diagrams and basins of attraction. The bifurcation analysis was supported by a path following method. The influence of each system parameter (except gap) on the system dynamics was studied in detail. The bifurcations known as interior crisis and boundary crisis were observed and discussed in this work. Notably, the parameter regions where various types of grazing induced bifurcations occurred were detected and investigated.

Keywords: Non-smooth systems; Backlash; Clearance; Impacts; Numerical simulation; Path following; Bifurcation analysis.
\end{abstract}

\section{Introduction}

Mathematical models of non-smooth systems have been extensively studied over the past decades by both analytical and numerical methods. These studies have shown a rich dynamical behaviour. Using mapping techniques, Shaw and Holmes [2] investigated a periodically forced single degree-of-freedom piecewise linear oscillator with the discontinuity in the restoring force. By implementing the impact rule, the case of rigid impact was also analysed. The oscillator with a backlash or a play was studied at the same time by Li et al. [3] and by Kleczka et al. [4], where the former work used the rigid constraint

Email addresses: a.chong@abdn.ac.uk (Antonio S.E. Chong), leyuan2003@sina.com (Yuan Yue), e.pavlovskaia@abdn.ac.uk (Ekaterina Pavlovskaia), m.wiercigroch@abdn.ac.uk (Marian Wiercigroch) 
approach. The latter paper uses the model considered in the present work, which has drawn an attention due to sudden unexpected changes of the chaotic dynamics referred to as crisis, using foundations of the works by Grebogi et al. [5] and Ueda [6], in which the collision of an unstable periodic orbit and a co-existing chaotic attractor is shown to cause the crisis phenomena. For the same system of [4], Luo et al. [7] numerically investigated the global chaos, whereas Wiercigroch [8] provided a further overview of the system dynamics through codimension-1 bifurcation diagrams. In addition, the analysed model is relevant to those to investigate gear-pair systems with a backlash, for which description with some analysis can be found in the works of Kaharaman et al. [9], Theodossiades et al. [10] and de Souza et al. [11]. Irregular responses, including crises and intermittent chaos were presented and discussed therein. An oscillator with a symmetrical trilinear spring and subjected to harmonic excitation was considered by Natsiavas [12], where an exact solution for periodic symmetric responses was obtained and its stability analysis was also undertaken.

To analyse the motion undergoing grazing impacts, Nordmark [13] studied the non-periodic motion caused by grazing incidence in an oscillator excited by a periodic force and subjected to a rigid amplitude constraint. He has shown that the discrete mapping in the neighbourhood of the grazing condition contains a square-root term, which gives rise to the so-called square-root singularity. In [14], Dankowicz and Nordmark developed a local analysis technique named discontinuity mapping to understand and explain the local behaviour of a model with stick-slip oscillations in the vicinity of grazing bifurcations. For systems with discontinuities in the vector field, this technique serves as a general method for stability and bifurcation analysis. The mapping introduced by Nordmark was reconsidered by Molenaar et al. [15] to derive grazing impact mappings caused by both rigid and elastic restraints. From the obtained maps, which introduced a few changes, the authors concluded that the square-root singularity persists in the systems with rigid restraints, whereas a $\frac{3}{2}$ singularity is involved in those with elastic restraints. In [16], Dankowicz and Zhao used the concept of discontinuity mapping to present a theoretical analysis of three codimension-one transitions scenarios associated with grazing conditions for a periodic response of an impact microactuator.

Another group of bifurcations that are unique to piecewise smooth systems are the so-called bordercollision bifurcations and which take place in iterative maps. Classification of all the possible bordercollision bifurcation scenarios can be found in works by di Bernardo et al. [17], where theoretical predictions applied to one and two-dimensional maps are presented to be in a good agreement with numerical simulations. Nusse and Yorke [18] also worked on these bifurcations, studying two-dimensional piecewise smooth maps and providing a general criterion for the ocurrence of such bifurcations. Moreover, a clas- 
sification of these bifurcations in system described by two-dimensional iterative maps was presented by Banerjee and Grebogi in [19].

Using the concept of the so-called discontinuity mapping, di Bernardo et al. in [20] and [21] presented a unified framework for grazing and sliding bifurcations in $n$-dimensional piecewise smooth dynamical systems of ODE's by deriving their normal form Poincaré maps. For the case of grazing bifurcations, it was shown that the map contains a square-root singularity where the flow is discontinuous at the grazing point, whereas a $\frac{3}{2}$ singularity otherwise. Therefore, rigid impact oscillators modelled with a restitution law belong to the first group while elastic impact oscillators where the impact is modelled with an unstressed spring belong to the second one. Impacting systems between these two types, in which the impacting wall is cushioned with a spring-damper support, have been studied by Ma et al. [22]. Here, numerical investigations demonstrated that for such a system the trace of the Jacobian matrix exhibits a square-root singularity at the grazing point whereas its determinant remains invariant. Furthermore, in [23] the effects of the individual components of the cushion on the character of the normal from of the map in and away from the immediate vicinity of the grazing orbit were numerically and experimentally investigated. Specifically, for four system configuration, it is probed how the trace and determinant vary as a non-impacting orbit is driven to impact through continuous change of a system parameter.

Among other important experimental investigations concerned with grazing events are those conducted by Stensson et al. [24] (for a spring-mass system impacting instantaneously with a moving base), by Piiroinen et al. [25] (for a single-degree-of-freedom horizontally excited pendulum that impacts with a rigid stop at a fixed angle) and by the Centre of Applied Dynamics Research at the University of Aberdeen [26-29]. Further, in [28] was discovered a narrow band of chaos near the grazing condition and in [29] was displayed the rich dynamical behaviour of the oscillator close to grazing by analysing the bifurcations taking place and a large number of co-existing attractors.

Dynamical systems with a play are fundamental and have many practical applications (e.g. gear boxes), however there is a little nonlinear dynamical analysis showing the global behaviour and focus on new types of bifurcations occurring in non-smooth systems. The paper probes into these areas and provides for the first time a global overview of the dynamic behaviour where all control system parameters except the gap were used. The paper also touches upon grazing and crisis bifurcations.

The layout of this paper is as follows. Section 2 presents the physical model and equation of motion of the considered system in detail. The analysis of the system dynamics is undertaken by direct numerical simulations and numerical continuation in Section 3, allowing each parameter of the system except the gap to vary in key ranges. Finally, closing remarks and future work discussion are given in Section 4 . 


\section{Mathematical modelling}

The physical model of the system under investigation is shown in Fig. 1(a). It is an oscillator of one degree-of-freedom, with piecewise linear restoring force characteristics depicted in Fig. 1(b) and excited by a harmonic external force of amplitude $A$ and frequency $\Omega$. The mass $m$ is attached to a linear damper with damping coefficient $c$ and a linear spring of stiffness $k$ acting every time the amplitude of the oscillations exceeds a gap $G$.

(a)

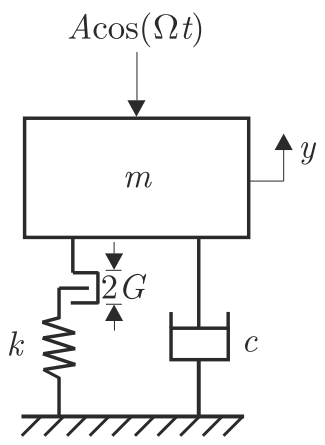

(b)

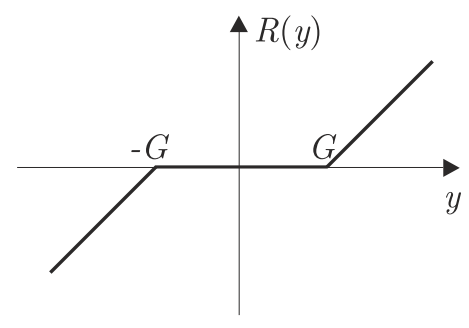

Figure 1: (a) Physical model and (b) restoring force characteristics of the considered oscillator with a play.

The motion of the system is governed by the piecewise linear equation

$$
m \ddot{y}+c \dot{y}+R(y)=A \cos (\Omega t),
$$

where the restoring force $R(y)$ reads as

$$
R(y)=\left\{\begin{array}{ccc}
k(y-G), & y>G \\
0 & , & |y| \leq G \\
k(y+G) & , & y<-G
\end{array}\right.
$$

By using a reference displacement $y_{0}$, the non-dimensional displacement $x=\frac{y}{y_{0}}$ and time $\tau=\omega_{n} t$ are defined, where $\omega_{n}=\sqrt{\frac{k}{m}}$ is the natural frequency. The non-dimensional parameters are also defined as

$$
\xi=\frac{c}{2 m \omega_{n}}, e=\frac{G}{y_{0}}, a=\frac{A}{y_{0} k}, \omega=\frac{\Omega}{\omega_{n}},
$$

where, $\xi$ is the damping ratio, $e$ is the non-dimensional gap, $a$ is the non-dimensional forcing amplitude and $\omega$ is the non-dimensional forcing frequency. Then, the equation of motion in the non-dimensional form is given as

$$
x^{\prime \prime}+2 \xi x^{\prime}+F_{r}(x)=a \cos (\omega \tau) .
$$


Here' stands for differentiation with respect to the nondimensional time $\tau$. $F_{r}(x)$ standing for the non-dimensional restoring force is given by

$$
F_{r}(x)=\left\{\begin{array}{cc}
x-e & , \quad x>e \\
0, & , \quad|x| \leq e \\
x+e & , \quad x<-e
\end{array}\right.
$$

The system vector field in non-autonomous and autonomous form is presented below in equations (6) and (7) respectively. For the latter, $s=\omega \tau \bmod 2 \pi$ defines an angular variable.

$$
\begin{aligned}
& \left\{\begin{array}{ccc}
x^{\prime} & = & v \\
v^{\prime} & = & a \cos (\omega \tau)-2 \xi v-F_{r}(x)
\end{array}\right. \\
& \left\{\begin{array}{ccc}
x^{\prime} & = & v \\
v^{\prime} & = & a \cos (s)-2 \xi v-F_{r}(x) \\
s^{\prime} & = & \omega
\end{array}\right.
\end{aligned}
$$

\section{Global dynamics evaluated by direct numerical simulations and numerical continuation}

Direct numerical simulations presented in this work were performed using our newly developed inhouse Matlab-based computational suite for non-smooth systems, ABESPOL [1]. To run numerical continuations a special interface has been created to link ABESPOL with the continuation core COCO [30]. In a user-friendly manner, this interface allows us to utilise existing general-purpose routines supplied by $\mathrm{COCO}$ for continuation and bifurcation analysis of smooth and non-smooth dynamical systems.

To run direct numerical simulations, ABESPOL used the Matlab implicit variable-step variable-order solver ode15s. This is a multistep solver based on numerical differentiation formulas of orders 1 to 5 . The default maximun order of 5 was used as well as relative and absolute tolerances of 1e-7 and 1e-9 respectively. Detection of events was done by the zero crossing functionality of this Matlab's solver, which relies on sign crossings between steps. The accuracy of event detections using Matlab's functionality is as close as possible to the precision of the computer, eps $\approx 2.2204 e-16$. To compute bifurcation diagrams, 200 first periods were disregarded to allow steady state responses to develop and 100 periods were recorded afterwards for periodic responses. For chaotic motions on Poincaré sections, 4900 extra periods were recorded.

To handle non-smooth systems when running direct numerical simulations in ABESPOL, the conceptual model set out by Wiercigroch in [31] was adopted. Such model is based on both splitting the global phase space into local subspaces where the system is assumed to be smooth, and considering each intersection between subspaces as a hypersurface where discontinuity events occur. To define these systems 
in ABESPOL, we employ the philosophy of the driver TC-HAT [32], which follows the model described in [31]. Hence, implementation of ABESPOL's routines for direct numerical simulations are based on defining two sets, namely, a set of operation modes containing smooth vector fields of local subspaces and a set of discontinuity events. Each event is made up of three components, that is, a pair with input and output modes $\left(m_{\text {in }}, m_{\text {out }}\right)$, a smooth scalar event function, $h$, to locate the event and a smooth jump function, $j$, that gives the output mode's initial point in terms of the input mode's final point.

For the analysed oscillator, let $\mathbf{V}:=[x, v, s]^{T} \in \mathbb{R}^{2} \times S^{1}$ be the state variables and $\mathbf{p}:=[a, \omega, \xi, e]^{T} \in$ $\left(\mathbb{R}^{+}\right)^{4}$ be the parameter's vector of the system. The phase space is split into the three local subspaces: $X_{1}$ for $|x| \leq e ; X_{2}$ for $x>e$; and $X_{3}$ for $x<e$. With this, the system can operate in the following three modes, where the motion is governed by the specified smooth vector fields:

No-Contact (NC), for the subspace $X_{1}$ :

$$
\mathbf{V}^{\prime}=f_{N C}(\mathbf{V}, \mathbf{p}):=\left(\begin{array}{ccc}
x^{\prime} & = & v \\
v^{\prime} & = & a \cos (s)-2 \xi v \\
s^{\prime} & = & \omega
\end{array}\right),
$$

Contact-Up (CU), for the subspace $X_{2}$ :

$$
\mathbf{V}^{\prime}=f_{C U}(\mathbf{V}, \mathbf{p}):=\left(\begin{array}{llc}
x^{\prime} & = & v \\
v^{\prime} & = & a \cos (s)-2 \xi v-x+e \\
s^{\prime}= & \omega
\end{array}\right),
$$

Contact-Down (CD), for the subspace $X_{3}$ :

$$
\mathbf{V}^{\prime}=f_{C D}(\mathbf{V}, \mathbf{p}):=\left(\begin{array}{llc}
x^{\prime} & = & v \\
v^{\prime} & = & a \cos (s)-2 \xi v-x-e \\
s^{\prime}= & \omega
\end{array}\right) .
$$

The discontinuity events take place on the discontinuity boundaries $\Sigma_{1}, \Sigma_{2}, \Sigma_{3}, \Sigma_{4}, \Sigma_{5}$ defined as

$$
\begin{aligned}
& \Sigma_{1}=\left\{\left(s_{i}, x_{i}, v_{i}\right): x_{i}=e, v_{i}>0, s_{i} \in[0,2 \pi)\right\}, \\
& \Sigma_{2}=\left\{\left(s_{i}, x_{i}, v_{i}\right): x_{i}=e, v_{i}<0, s_{i} \in[0,2 \pi)\right\}, \\
& \Sigma_{3}=\left\{\left(s_{i}, x_{i}, v_{i}\right): x_{i}=-e, v_{i}>0, s_{i} \in[0,2 \pi)\right\}, \\
& \Sigma_{4}=\left\{\left(s_{i}, x_{i}, v_{i}\right): x_{i}=-e, v_{i}<0, s_{i} \in[0,2 \pi)\right\}, \\
& \Sigma_{5}=\left\{\left(s_{i}, x_{i}, v_{i}\right): x_{i} \in \mathbb{R}, v_{i} \in \mathbb{R}, s_{i}=2 \pi\right\},
\end{aligned}
$$

where $\Sigma_{5}$ is defined to limit the angular variable $s$ to the interval $[0,2 \pi)$. The events $\operatorname{Ev}_{1}, \ldots, \operatorname{Ev}_{7}$ shown in Table 1 are defined for a direct numerical simulation to be carried out in ABESPOL. The additional events $\mathrm{Ev}_{8}, \ldots, \mathrm{Ev}_{11}$, corresponding to grazing contacts with the curves $x-e=0$ and $x+e=0$, are defined for the purpose of numerical continuations supported by COCO. 
Table 1: Events of the system, such that the last four are only defined for numerical continuations.

\begin{tabular}{|c|c|c|c|c|c|}
\hline Event Index & Event Name & Mode in $\left(m_{i n}\right)$ & Mode out $\left(m_{\text {out }}\right)$ & Event function $(h(\mathbf{V}, \mathbf{p}))$ & Jump function $(j(\mathbf{V}))$ \\
\hline $\mathrm{Ev}_{1}$ & $\mathrm{NC} 2 \mathrm{CU}$ & $\mathrm{NC}$ & $\mathrm{CU}$ & $h_{C U}:=x-e$ with $\dot{x}>0$ & $j_{i d}:=\mathbf{V}$ \\
\hline $\mathrm{Ev}_{2}$ & $\mathrm{NC} 2 \mathrm{CD}$ & $\mathrm{NC}$ & $\mathrm{CD}$ & $h_{C D}:=x+e$ with $\dot{x}<0$ & $j_{i d}:=\mathbf{V}$ \\
\hline $\mathrm{Ev}_{3}$ & $\mathrm{NC} 2 \pi$ & $\mathrm{NC}$ & $\mathrm{NC}$ & $h_{2 \pi}:=s-2 \pi$ with $\dot{s}>0$ & $j_{2 \pi}:=[x, v, s-2 \pi]^{T}$ \\
\hline $\mathrm{Ev}_{4}$ & $\mathrm{CU} 2 \mathrm{NC}$ & $\mathrm{CU}$ & $\mathrm{NC}$ & $h_{C U}:=x-e$ with $\dot{x}<0$ & $j_{i d}:=\mathbf{V}$ \\
\hline $\mathrm{Ev}_{5}$ & $\mathrm{CU} 2 \pi$ & $\mathrm{CU}$ & $\mathrm{CU}$ & $h_{2 \pi}:=s-2 \pi$ with $\dot{s}>0$ & $j_{2 \pi}:=[x, v, s-2 \pi]^{T}$ \\
\hline $\mathrm{Ev}_{6}$ & $\mathrm{CD} 2 \mathrm{NC}$ & $\mathrm{CD}$ & $\mathrm{NC}$ & $h_{C D}:=x+e$ with $\dot{x}>0$ & $j_{i d}:=\mathbf{V}$ \\
\hline $\mathrm{Ev}_{7}$ & $\mathrm{CD} 2 \pi$ & $\mathrm{CD}$ & $\mathrm{CD}$ & $h_{2 \pi}:=s-2 \pi$ with $\dot{s}>0$ & $j_{2 \pi}:=[x, v, s-2 \pi]^{T}$ \\
\hline $\mathrm{Ev}_{8}$ & GRUinNC & $\mathrm{NC}$ & $\mathrm{NC}$ & $h_{G R}:=v$ with $\dot{v}<0$ & $j_{i d}:=\mathbf{V}$ \\
\hline $\mathrm{Ev}_{9}$ & GRDinNC & $\mathrm{NC}$ & $\mathrm{NC}$ & $h_{G R}:=v$ with $\dot{v}>0$ & $j_{i d}:=\mathbf{V}$ \\
\hline $\mathrm{Ev}_{10}$ & GRinCU & $\mathrm{CU}$ & $\mathrm{CU}$ & $h_{G R}:=v$ with $\dot{v}>0$ & $j_{i d}:=\mathbf{V}$ \\
\hline $\mathrm{Ev}_{11}$ & GRinCD & $\mathrm{CD}$ & $\mathrm{CD}$ & $h_{G R}:=v$ with $\dot{v}<0$ & $j_{i d}:=\mathbf{V}$ \\
\hline
\end{tabular}

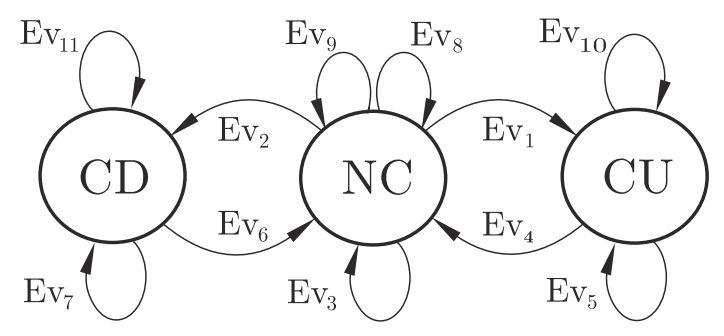

Figure 2: Operation modes and event of the system. Details of each event are given in Table 1.

With regard to the numerical continuation, orbits must be defined as a sequence of modes and events or alternatively as a sequence of segments given by a vector field in an output operation mode and one of its possible subsequent events. From Table 1, segments can be defined as shown in Table 2. For simplicity, segment names are the same of their associated events.

Table 2: Segments defined for numerical continuation through the interface that links ABESPOL [1] and COCO [30].

\begin{tabular}{cccc}
\hline Segment Index & Segment Name & Vector field & Event Index \\
\hline$I_{1}$ & NC2CU & $f_{N C}$ & $\mathrm{Ev}_{1}$ \\
$I_{2}$ & $\mathrm{NC} 2 \mathrm{CD}$ & $f_{N C}$ & $\mathrm{Ev}_{2}$ \\
$I_{3}$ & $\mathrm{NC} 2 \pi$ & $f_{N C}$ & $\mathrm{Ev}_{3}$ \\
$I_{4}$ & $\mathrm{CU} 2 \mathrm{NC}$ & $f_{C U}$ & $\mathrm{Ev}_{4}$ \\
$I_{5}$ & $\mathrm{CU} 2 \pi$ & $f_{C U}$ & $\mathrm{Ev}_{5}$ \\
$I_{6}$ & $\mathrm{CD} 2 \mathrm{NC}$ & $f_{C D}$ & $\mathrm{Ev}_{6}$ \\
$I_{7}$ & $\mathrm{CD} 2 \pi$ & $f_{C D}$ & $\mathrm{Ev}_{7}$ \\
$I_{8}$ & GRUinNC & $f_{N C}$ & $\mathrm{Ev}_{8}$ \\
$I_{9}$ & GRDinNC & $f_{N C}$ & $\mathrm{Ev}_{9}$ \\
$I_{10}$ & GRinCU & $f_{C U}$ & $\mathrm{Ev}_{10}$ \\
$I_{11}$ & GRinCD & $f_{C D}$ & $\mathrm{Ev}_{11}$ \\
\hline
\end{tabular}




\subsection{Influence of the forcing amplitude}

Bifurcation diagrams as a function of the forcing amplitude are shown in Figs 3 and 4, in which the control parameter ranges from 0.01 to 1.30 . Two forcing frequencies were analysed, $\omega=0.1$ and $\omega=1.0$, with the damping ratio and the gap fixed to $\xi=0.02$ and $e=1.0$ respectively.

Fig. 3 presents the bifurcation diagram calculated for $\omega=0.1$ where additional panels demonstrate the trajectories and Poincaré maps for the selected values of the external force amplitude. In those graphs, magenta vertical lines denote the discontinuity boundaries and the left line corresponds to the ContactDown - No-Contact modes boundary whereas the right line corresponds to No-Contact - Contact-Up modes boundary. The parts of the trajectories belonging to the No-Contact mode are shown in green and the parts belonging to Contact-Down and Contact-Up modes are in blue. As can be seen from this figure, the shape of the periodic solution vary significantly and the obtained periodic responses might have different number of contacts with both discontinuity boundaries. Therefore to classify the obtained periodic responses we will introduce the following notations: each periodic response will be characterised by three integer numbers, i.e. period- $(\mathrm{n}, \mathrm{m}, \mathrm{k})$ where $n$ will represent the time duration of solution, and $m$ and $k$ will indicate the numbers of crossing to the Contact-Down mode and crossing to the Contact-Up mode, respectively. For example, the response shown in Fig. 3(c) for $a=0.12352$ is period-(1,3,3) as the period of this response is equal to the period of the external excitation and there are three impacts with the left and three impacts with the right boundaries. In addition, for the classification of the responses, the term symmetric is used for those responses whose trajectory has rotational symmetry with respect to the origin of coordinates of the $(x, v)$ plane, and the term asymmetric otherwise.

As can be seen from Fig. 3, with the increase of the external force amplitude, a series of periodic attractors separated by windows of chaotic responses were recorded. They range from symmetric period$(1,1,1)$ response at $a \in(0.01000,0.01258)$ and $(1.19680,1.30000)$ (see Fig. 3(1)), period- $(1,2,2)$ response at $a \in(0.03580,0.04612)$ and $(1.16840,1.19680)$, period-(1,3,3) response shown in Fig. 3(c) at $a \in$ $(0.11062,0.12868)$ and in Fig. $3(\mathrm{k})$ at $a \in(1.14780,1.16840)$, and period- $(1,4,4)$ response shown in Fig. 3(e) at $a \in(0.27574,0.32218)$ to asymmetric period- $(1,2,2)$ response at $a \in(0.04612,0.05386)$, period-(1,3,3) response at $a \in(0.12868,0.13900)$, period- $(1,4,4)$ response at $a \in(0.32218,0.34024)$ and period- $(1,4,3)$ response at $a \in(0.46924,0.49246)$. Asymmetric period- $(2,2,2)$ attractors were found at $a \in(0.01258,0.01774)$ and period- $(2,8,8)$ attractors at $a \in(0.34024,0.34798)$ together with period- $(2,8,6)$ response shown in Fig. 3(g) at $a \in(0.49246,0.50278)$. Symmetric period- $(3,8,8)$ attractors shown in Fig. 3(i) were obtained at $a \in(0.82270,0.88462)$ while asymmetric period- $(3,8,8)$ attractors appear at $a \in(0.80464,0.82270)$ and $(0.88462,0.89494)$. 


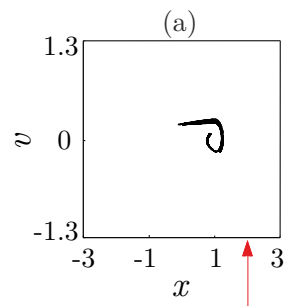

(b)

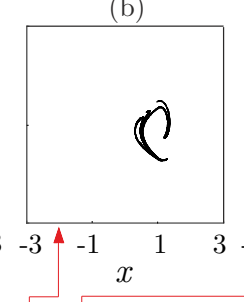

(d)
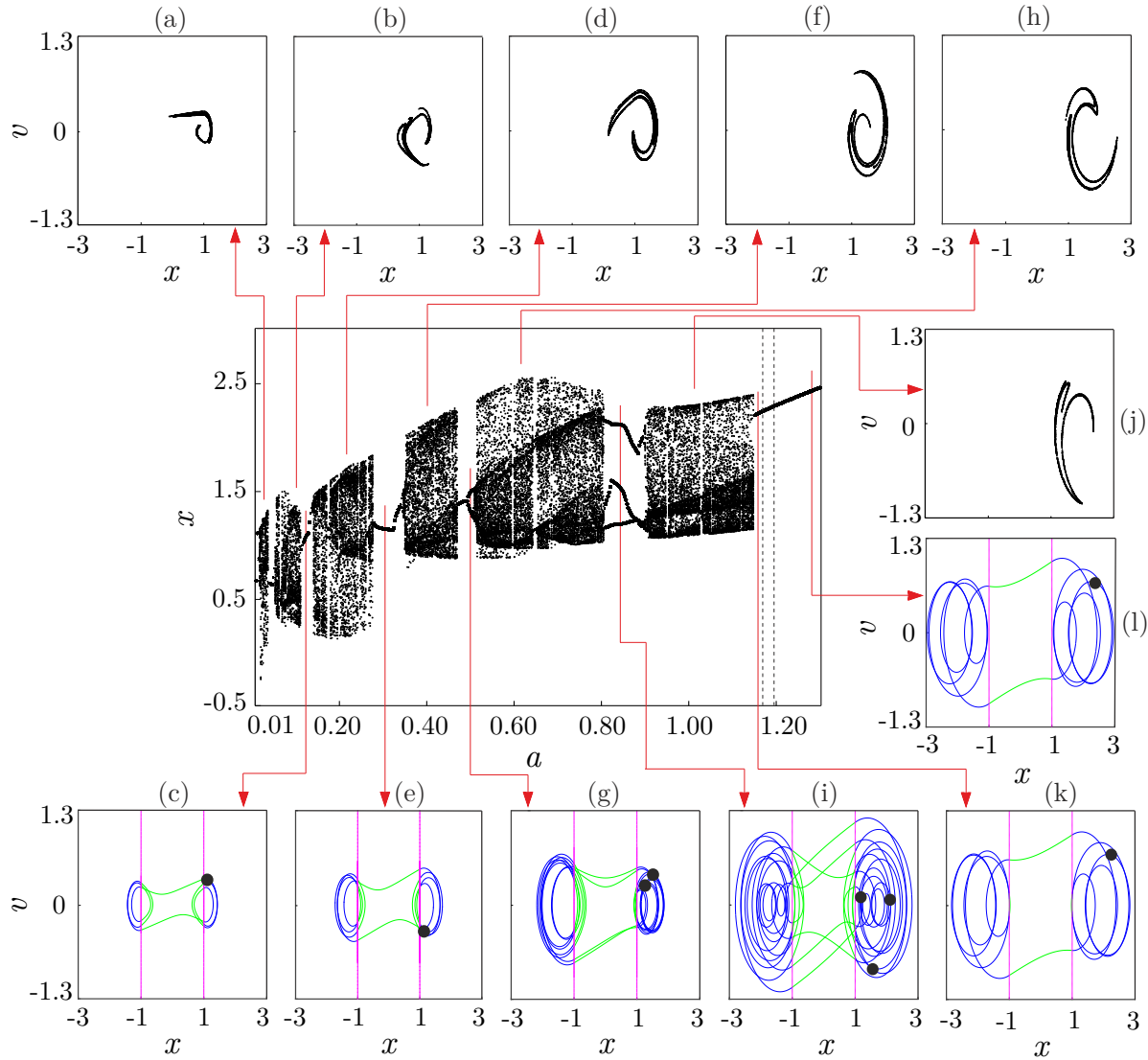

Figure 3: Bifurcation diagram of displacement as a function of the forcing amplitude, $a$, computed for $\xi=0.02, e=1$ and $\omega=0.1$, where the control parameter was increased. Dashed vertical lines stand for grazing incidence. Additional panels show trajectories and Poincaré maps on the $(x, v)$ planes computed for the following amplitudes: (a) 0.02806, (b) 0.09520, (c) 0.12352, ,d) 0.21382, (e) 0.30928 , (f) 0.39958, (g) 0.50020, (h) 0.62662, (i) 0.83818, (j) 1.09100 , (k) 1.16070 and (l) 1.27940. There, operation in No-Contact mode is in green, whereas operation in Contact-Up and Contact-Down is in blue; points of the Poincaré maps are in the same colours used in the bifurcation diagram; and magenta vertical lines denote the discontinuity boundaries.

At $a=0.34024$, we observe a period-doubling bifurcation where asymmetric period- $(1,4,4)$ response becomes asymmetric period- $(2,8,8)$ response. Similarly, at $a=0.49246$ a period-doubling bifurcation occurs creating an asymmetric period- $(2,8,6)$ response from an asymmetric period- $(1,4,3)$ response.

The evolution of chaotic attractors recorded at $a \in(0.01774,0.03580),(0.05386,0.11062),(0.13900,0.27574)$, $(0.34798,0.46924),(0.51052,0.80464)$ and $(0.90526,1.14780)$ is presented in Fig. 3(a), 3(b), 3(d), 3(f), $3(\mathrm{~h})$ and $3(\mathrm{j})$ calculated for $a=0.02806,0.09520,0.21382,0.39958,0.62662$, and 1.09100, respectively. As can be seen from this figure, we observe an increase of the chaotic attractor size with the increase in the excitation amplitude.

Our analysis also reveals that pitchfork bifurcations take place at $a=0.04612,0.12868,0.32218$, 0.82270 and 0.88462 , the period-doubling cascades are observed at $a \in(0.50278,0.51052)$ and $(0.89494,0.90526)$, and the grazing events occur at $a=1.16840$ and 1.19680 . 
Finally, it is worth noting narrow intervals of periodic responses observed in the chaotic motion windows. The examples are asymmetric period-(1,3,2) attractors at $a \in(0.06160,0.06676)$, period$(1,4,3)$ attractors at $a \in(0.16996,0.17254)$ and period- $(1,8,6)$ attractors at $a \in(0.17512,0.17770)$ as well as symmetric period- $(3,10,10)$ attractors at $a \in(0.59308,0.60082)$ and $(0.64210,0.65242)$.

The second bifurcation diagram calculated for the varying excitation amplitude is presented in Fig. 4 for $\omega=1.0$. As can be seen from this figure, much simpler behaviour is observed in the case of larger excitation frequency.

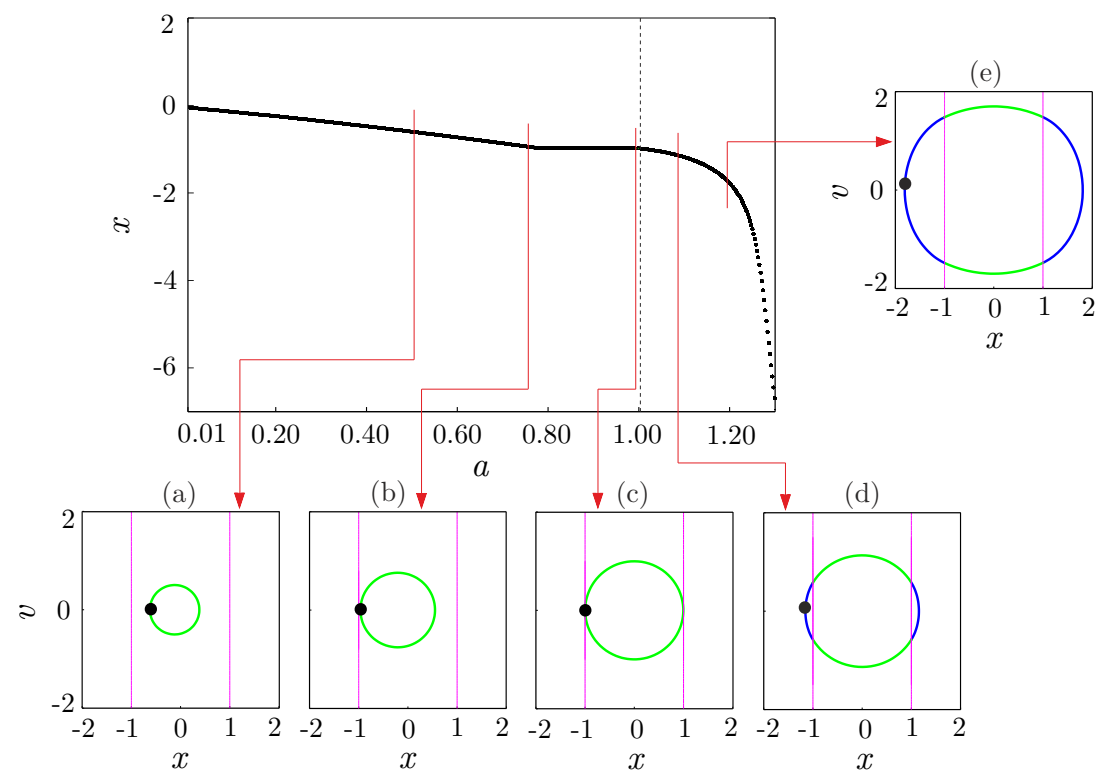

Figure 4: Bifurcation diagram of displacement as a function of the forcing amplitude, $a$, computed for $\xi=0.02, e=1$ and $\omega=1$, where the control parameter was increased. Dashed vertical lines stand for grazing incidence. Additional panels show trajectories and Poincaré maps on the $(x, v)$ planes computed for the following amplitudes: (a) 0.50278, (b) 0.75820, (c) 0.99814, (d) 1.08330 and (e) 1.19940. There, operation in No-Contact mode is in green, whereas operation in Contact-Up and Contact-Down is in blue; points of the Poincaré maps are in the same colours used in the bifurcation diagram; and magenta vertical lines denote the discontinuity boundaries.

As the amplitude increases, the non-impacting period- $(1,0,0)$ attractors are observed at $a \in(0.0100,1.0007)$. The trajectories and Poincaré maps of these responses are demonstrated in Fig. 4(a), 4(b) and 4(c). At around $a=1.00330$, a grazing incidence takes place giving birth to period- $(1,1,1)$ attractors and the typical examples are presented in Fig. 4(d) and 4(e). As can be seen from this figure, the amplitude of vibrations increases with the increase of the excitation amplitude and the maximum is observed at $a=1.30000$ in the considered range of the excitation amplitudes.

From the presented simulation results, it is clear that a number of distinct system responses was observed in the considered parameter window, $a \in(0.01,1.30)$, for the small value of $\omega=0.1$ whereas for $\omega=1.0$ only period- $(1,0,0)$ and period- $(1,1,1)$ responses were recorded. Our extended analysis of the system responses for the frequency values lying between 0.1 and 1.0 reveals that the chaotic responses are 
present in the system dynamics for $\omega \in(0.100,0.445)$, but as the frequency increases beyond this range, only periodic responses are observed.

\subsection{Influence of the damping ratio}

The bifurcation diagrams calculated with the damping ratio, $\xi$, as the control parameter are shown in Figs 5 and 6 . The interval $\xi \in(0.005,0.200)$ was analysed for two values of forcing amplitude, $a=0.1$ and $a=0.7$, and with the forcing frequency and the gap fixed to $\omega=0.3$ and $e=1$ respectively.

In Fig. 5, the bifurcation diagrams computed by direct numerical simulation for increasing and decreasing control parameter are overlaid with the unstable period- $(1,1,1)$ responses obtained by numerical continuation. The signature $\left\{I_{1}, I_{4}, I_{2}, I_{6}, I_{3}\right\}$, (see Table 2), was used for the continuation run, which was started at an orbit on the branch of the impacting period-(1,1,1) attractors.

As can be seen from this figure, for increasing damping ratio, symmetric period- $(1,1,1)$ attractors are observed for $\xi \in(0.00500,0.13778)$ and the typical responses are shown in Fig. 5(a) and Fig. 5(b). At $\xi=0.13778$, where a saddle-node (SN) bifurcation occurs, the system jumps to a non-impacting period-(1,0,0) attractor and its typical trajectories are shown in Fig. 5(f) and 5(g).

As we consider decreasing damping ratio from $\xi=0.20000$, an asymmetric non-impacting period$(1,0,0)$ attractor shown in Fig. $5(\mathrm{~g})$ for $\xi=0.19844$ turns into symmetric one as shown in Fig. $5(\mathrm{f})$ for $\xi=$ 0.13994 and subsequently turns into asymmetric orbit close to grazing incidence with the other boundary, Contact-Up, as shown in Fig. $5(\mathrm{e})$ for $\xi=0.12395$. Then, the orbit grows until it becomes symmetric again and comes into grazing contact (GR) with both Contact-Up and Contact-Down boundaries. This grazing event occurring around $\xi=0.072593$ is visible in the zoom-up window placed in the top righthand corner of the bifurcation diagram. After the grazing, impacting period-(1,1,1) attractor is observed between $\xi=0.072593$ and $\xi=0.072335$ where another saddle-node (SN) bifurcation occurs.

Using the continuation technique, an unstable symmetric impacting period- $(1,1,1)$ response is obtained at $\xi \in(0.072335,0.13778)$ which connect two presented saddle-node points. In this damping ratio range, two types of co-existing responses are observed. For damping ratios $\xi \in(0.072335,0.072593)$, three impacting period-(1,1,1) solutions co-exist, one of which is unstable; whereas for $\xi \in(0.072593,0.13778)$, there are two impacting period-(1,1,1) orbits (one of which is unstable) co-existing with a non-impacting period-(1,0,0) attractor. Fig. 5(c) demonstrates the sample trajectory of the unstable impacting period$(1,1,1)$ response for $\xi=0.07754$ whereas Fig. $5(\mathrm{~d})$ shows the trajectory of stable co-existing non-impacting period-(1,0,0) response at the same damping ratio. 


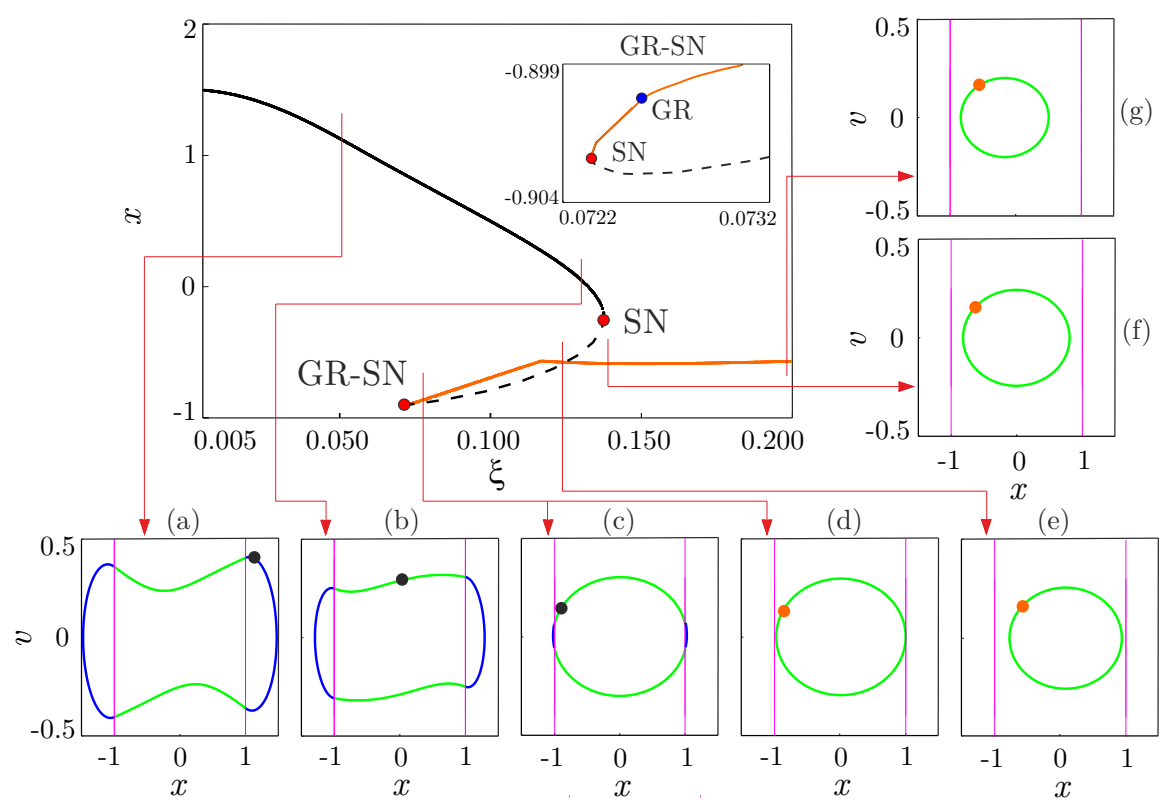

Figure 5: Bifurcation diagram of displacement as a function of the damping ratio, $\xi$, computed for $\omega=0.3, e=1$ and $a=0.1$, where the control parameter was increased and decreased. To detect unstable orbits, numerical continuation was employed. Co-existing attractors are shown in black and orange, and stable and unstable periodic orbits are presented by solid and dashed lines respectively. Labels SN and GR denote saddle-node bifurcation points and grazing event respectively. Additional panels show trajectories and Poincaré maps on the $(x, v)$ planes computed for the following damping ratios: (a) 0.05063, (b) 0.13058, (c) 0.07754, (d) 0.07754, (e) 0.12395, (f) 0.13994 and (g) 0.19844 . There, operation in No-Contact mode is in green, whereas in Contact-Up and Contact-Down is in blue; points of the Poincare maps are in the same colours used in the bifurcation diagram; and magenta vertical lines denote the discontinuity boundaries.

In Fig. 6, the bifurcation diagrams computed by direct numerical simulation by increasing and decreasing damping ratio are presented for $a=0.7$. As can be seen from this figure, two asymmetric period-(1,1,1) attractors depicted by black and orange points co-exist for $\xi \in(0.00500,0.01514)$, and typical trajectories of these attractors are shown in Fig. 6(a) and 6(b) for $\xi=0.01085$. Both of these attractors undergo period-doubling bifurcations $(\mathrm{PD})$ at $\xi=0.01514$ and a typical trajectory of period$(2,2,2)$ black attractor is presented in Fig. 6(c). As the damping ratio increases further, the system response turns to chaotic at $\xi=0.02489$, and this chaotic motion shown in Fig. 6(d) persists until 0.05234. Fig. 7 provides a detailed bifurcation diagram showing co-existing solutions and discontinuous bifurcations of a region around this value of the damping ratio, and a typical trajectory of the symmetric solution in such region, period-(3,4,4) response, is shown in Fig. 6(e). 


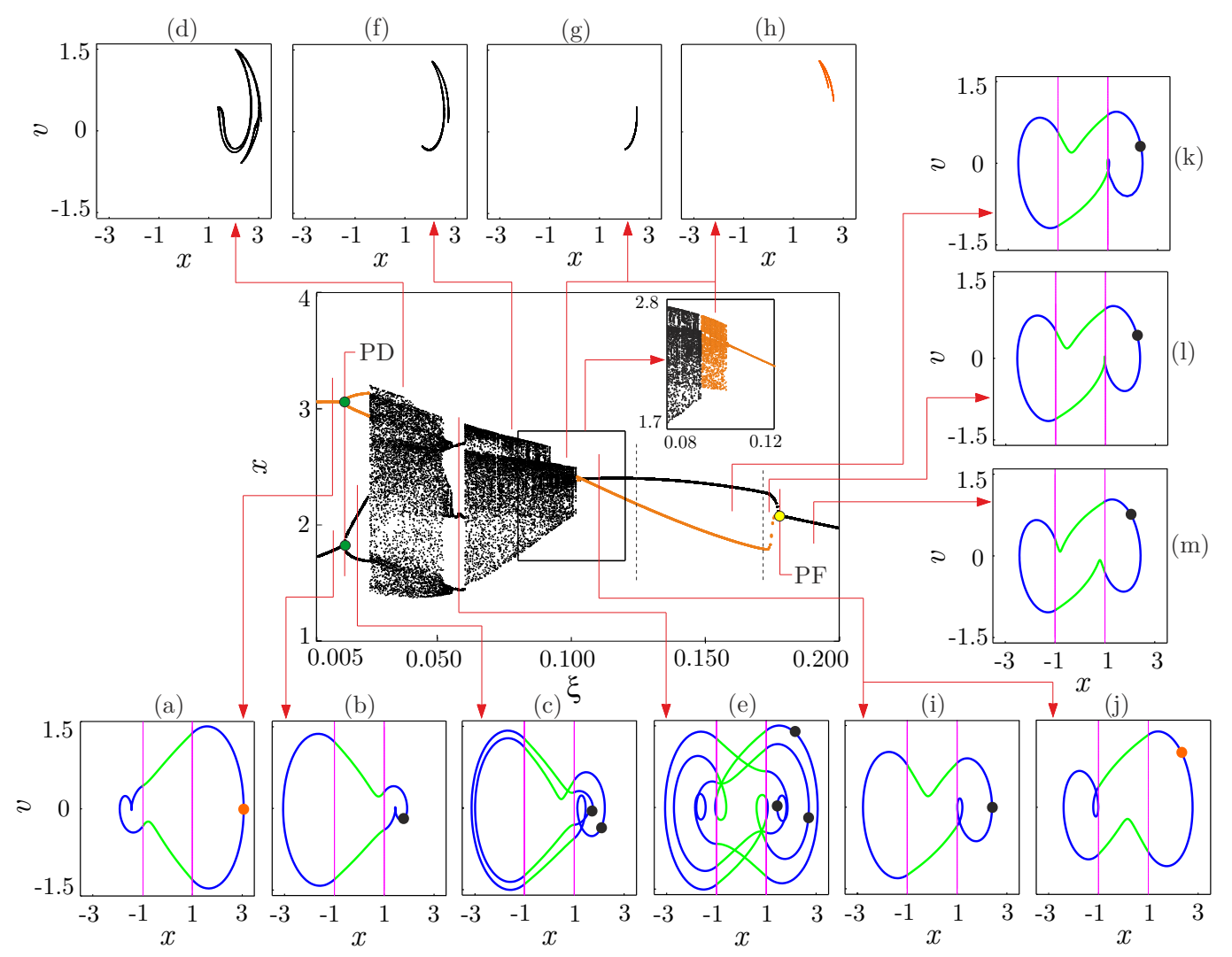

Figure 6: Bifurcation diagram of displacement as a function of the damping ratio, $\xi$, computed for $\omega=0.3, e=1$ and $a=0.7$, where the control parameter was increased and decreased. Co-existing attractors are shown in black and orange. Labels $\mathrm{SN}, \mathrm{PD}$ and PF denote saddle-node, period-doubling and pitchfork bifurcation points. Grazing events are represented by dashed vertical lines. Additional panels show trajectories and Poincaré maps on the $(x, v)$ planes computed for the following damping ratios: (a) 0.01085, (b) 0.01085, (c) 0.02021, (d) 0.03698, (e) 0.05804, (f) 0.07715, (g) 0.09821, (h) 0.09821, (i) $0.11030,(\mathrm{j})$ 0.11030, (k) 0.16022, (l) 0.17387 and $(\mathrm{m})$ 0.19064. There, operation in No-Contact mode is in green, whereas in Contact-Up and Contact-Up-Down is in blue; points of the Poincaré maps are in the same colours used in the bifurcation diagram; and magenta vertical lines denote the discontinuity boundaries.

Another window of the chaotic response is observed at $\xi \in(0.06038,0.09197)$, and the Poincaré map of the chaotic attractor from this range is demonstrated in Fig. 6(f) computed for $\xi=0.07715$. For $\xi \in(0.09197,0.10211)$ two chaotic attractors co-exist and the second one is shown by orange points in the zoom-up window placed at the top right-hand side of the bifurcation diagram, whereas the Poincaré maps of these co-existing chaotic attractors are presented in Fig. $6(\mathrm{~g})$ and $6(\mathrm{~h})$ for $\xi=0.09821$.

As can be seen from this figure, two co-existing asymmetric period- $(1,1,1)$ attractors are observed at $\xi \in(0.10211,0.17777)$, and their trajectories are shown in Fig. $6(\mathrm{i})$ and $6(\mathrm{j})$ for $\xi=0.11030$. Two grazing incidences occur at around $\xi=0.12376$ and 0.17114 and they are marked by the dashed vertical lines in the bifurcation diagram. Trajectories computed for $\xi=0.11030,0.16022$ and 0.17387 , and shown in Fig. $6(\mathrm{i}), 6(\mathrm{k})$ and $6(\mathrm{l})$, respectively, demonstrate the difference in the obtained responses as period- $(1,1,1)$ response becomes period- $(1,1,2)$ one at the first grazing event and goes back to period- $(1,1,1)$ orbit at the 
second grazing event. A pitchfork bifurcation $(\mathrm{PF})$ takes place at 0.17777 , where the asymmetric orbits merge giving birth to a symmetric period- $(1,1,1)$ attractor shown in Fig. $6(\mathrm{~m})$ for $\xi=0.19064$ which is recorded until the end of the analysed interval.

It should be noted that in the analysed interval, $\xi \in(0.005,0.200)$, only periodic behaviour was observed for $a=0.1$, whereas for $a=0.7$ there is a mixture of chaotic and periodic responses. Our additional simulations for $a \in(0.1,0.7)$ reveal that the chaotic responses are present in the system dynamics for the higher values of forcing amplitude at $a>0.179$.

To demonstrate the presence of two additional discontinuous bifurcations, specifically interior and boundary crisis, a detailed bifurcation diagram for $\xi \in(0.050,0.065)$ was constructed and it is presented in Fig. 7. Again, calculations for increasing and decreasing of the damping ratio were performed to record present co-existing solutions. It was found that interior crisis (IC), which is the finite enlargement of the chaotic attractor, occurs at damping ratios $\xi=0.05234$ and 0.05558 . Boundary crisis $(\mathrm{BC})$ taking place at 0.05861 corresponds to the disappearance of a chaotic attractor and its basin of attraction. A cascade of period-doubling bifurcations (PD) begins at 0.05657 for the decreasing control parameter. The co-existing attractors, depicted by black and orange points, are recorded in the intervals $\xi \in(0.05558,0.05801)$ and $(0.05861,0.06032)$. As the control parameter is increased, the first interval ends with pitchfork bifurcation $(\mathrm{PF})$ and the second ends with saddle-node bifurcation (SN).

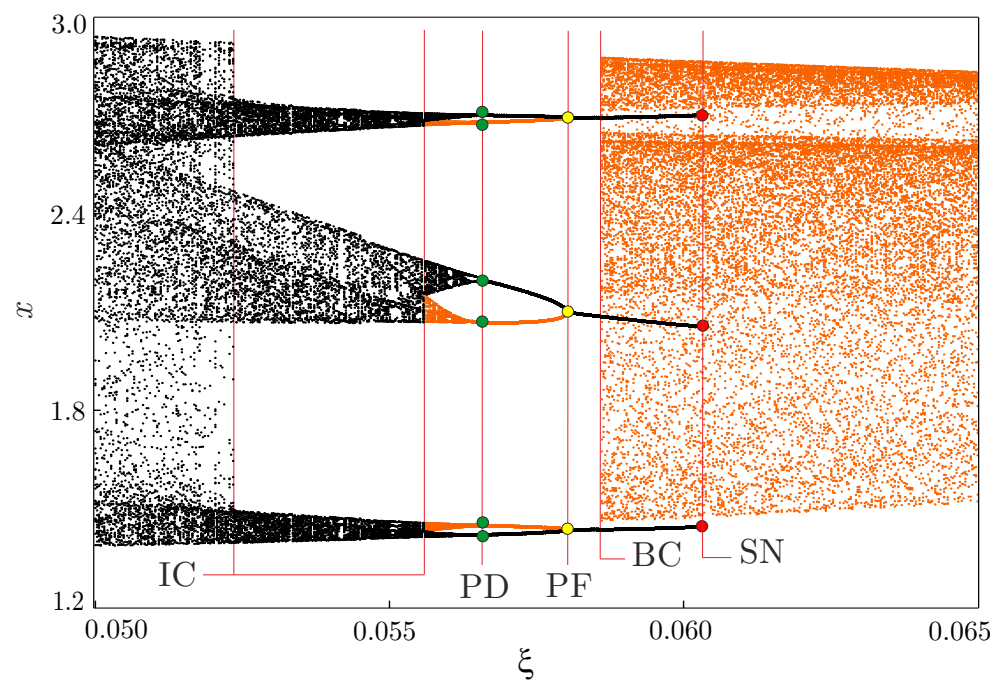

Figure 7: Bifurcation diagram of displacement as a function of the damping ratio, $\xi$, computed for $\omega=0.3, e=1$ and $a=0.7$, where the control parameter was increased and decreased. This figure provides insight into a region of the Fig. 6. Co-existing attractors are shown in black and orange. Labels SN, PD and PF denote saddle-node, period-doubling and pitchfork bifurcation points. Labels IC and BC mark the onset of interior crisis and boundary crisis respectively. 


\subsection{Influence of the forcing frequency}

Bifurcation diagrams shown in Figs 8 and 9 present the dynamics of the system under varying the forcing frequency, $\omega$, from 0.01 to 1.10 . The results are obtained for two values of damping ratio, $\xi=0.02$ and $\xi=0.20$ with the forcing amplitude and the gap set to $a=0.3$, and $e=1$ respectively.

As can be seen from Fig. 8 calculated for small damping ratio $\xi=0.02$, at lower forcing frequency $\omega \in$ $(0.01000,0.34140)$ the system demonstrates a mixture of chaotic and periodic responses. The evolution of the chaotic responses is shown in Figs $8(\mathrm{a}), 8(\mathrm{~b}), 8(\mathrm{c}), 8(\mathrm{e}), 8(\mathrm{~h})$ and $8(\mathrm{k})$ where the grow of the attractors size and increasing complexity are presented.

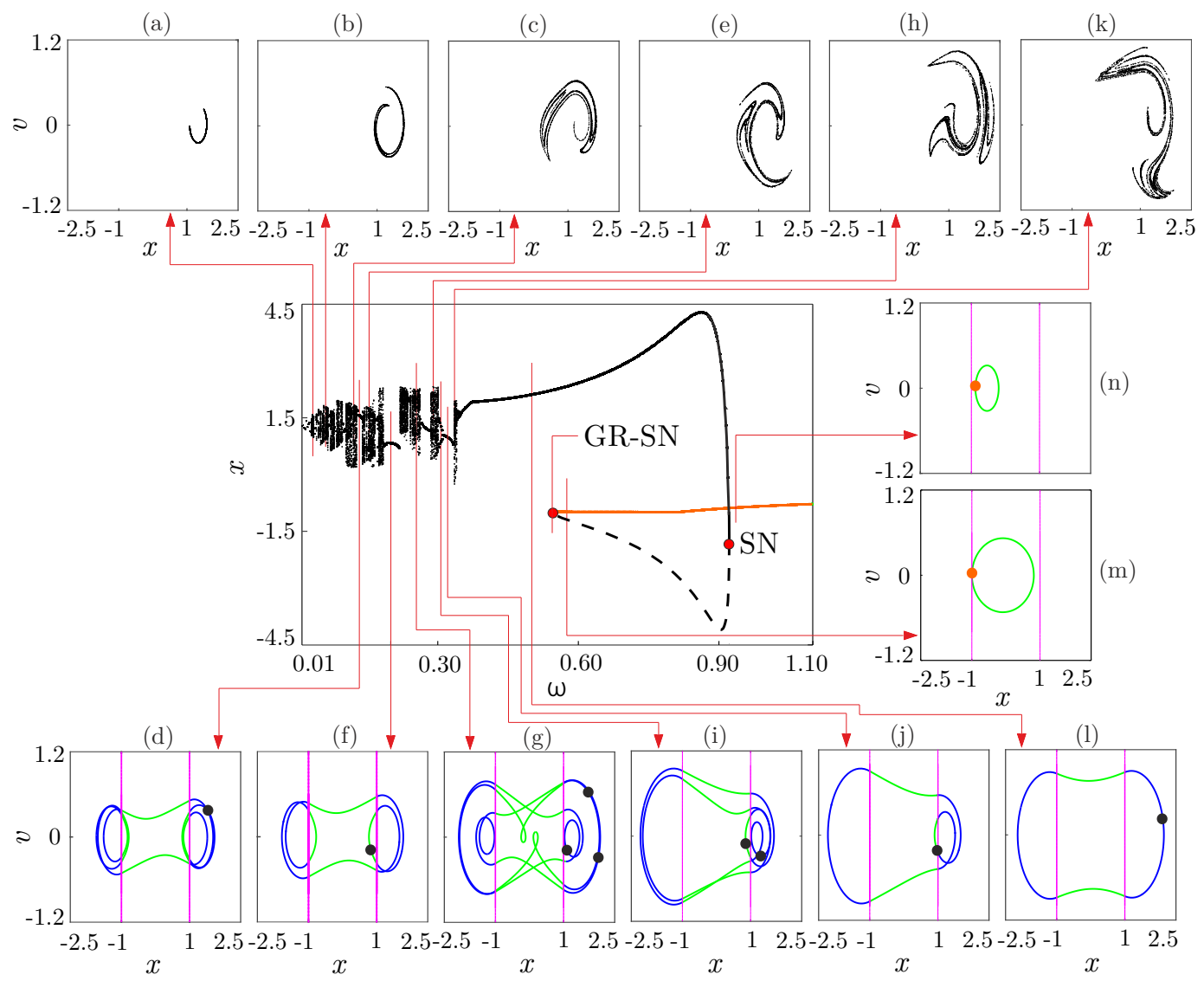

Figure 8: Bifurcation diagram of displacement as a function of the forcing frequency, $\omega$, computed for $a=0.3, e=1$ and $\xi=0.02$, where the control parameter was increased and decreased. To locate the unstable branch, numerical continuation was employed. Co-existing attractors are in black and orange, and stable and unstable periodic orbits are presented by solid and dashed lines respectively. Labels SN and GR denote saddle-node bifurcation points and grazing event respectively. Additional panels show trajectories and Poincaré maps on the $(x, v)$ planes computed for the following frequencies: (a) 0.03180, (b) 0.06014, (c) 0.12118, (d) 0.13208, (e) 0.15388, (f) 0.19966, (g) 0.25416, (h) 0.29122, (i) 0.30430, (j) 0.32174, (k) 0.33482 , (l) 0.50050, (m) 0.57391 and (n) 0.93650. There, operation in No-Contact mode is in green, whereas in Contact-Up and Contact-Down is in blue; points of the Poincaré maps are in the same colours used in the bifurcation diagram; and magenta vertical lines denote the discontinuity boundaries.

In between the chaotic windows, a number of periodic solutions is recorded and their trajectories are shown in Fig. 8(d) (period-(1,3,3) response at $\omega=0.13208)$, Fig. 8(f) (period-(1,2,2) response at 
$\omega=0.19966), 8(\mathrm{~g})$ (period- $(3,3,3)$ at $\omega=0.25416)$, Fig. $8(\mathrm{i})$ (period- $(2,2,4)$ at $\omega=0.30430)$ and Fig. $8(\mathrm{j})$ (period- $(1,1,2)$ at $\omega=0.32174)$. At 0.37842 , a pitchfork bifurcation takes place, and as the forcing frequency increases, a symmetric period-(1,1,1) response shown in Fig. $8(1)$ for $\omega=0.50050$ is observed until $\omega=0.92109$, where a saddle-node bifurcation (SN) occurs. At this frequency the solution jumps to a non-impacting asymmetric period-(1,0,0) attractor shown in Fig. 8(n) for $\omega=0.93650$, which is observed to the end of the parameter window where $\omega=1.1$.

Considering the decreasing forcing frequency, $\omega$, from the value of 1.1 , we observe the asymmetric period-(1,0,0) attractor (shown in Fig. 8(n) for $\omega=0.93650$ and in Fig. 8(m) for $\omega=0.57391$ ) up to $\omega=0.54664$, where the response turns into an impacting period- $(1,1,1)$ orbit at a grazing event (GR). Shortly after, there is a saddle-node bifurcation $(\mathrm{SN})$ at 0.54505 where the response jumps to period$(1,1,1)$ response described earlier. As with the behaviour observed in Fig. 5, an unstable period-(1,1,1) branch connects the SN points. To obtain this unstable period-(1,1,1) orbit by numerical continuation, the signature $\left\{I_{1}, I_{4}, I_{2}, I_{7}, I_{6}\right\}$ (see Table 2) was used. This computation was started at an orbit on the branch of the co-existing impacting period- $(1,1,1)$ attractors.

In Fig. 9, another bifurcation diagram is presented for a larger value of the damping ratio, $\xi=0.2$. This diagram presents results from direct numerical simulations, where the forcing frequency was increased and decreased, overlaid with the unstable period- $(1,1,1)$ responses obtained by numerical continuation. The signature $\left\{I_{1}, I_{4}, I_{2}, I_{6}, I_{3}\right\}$, (see Table 2), was used for the continuation run, which was started at an orbit on the branch of the impacting period- $(1,1,1)$ attractors.

As observed in this figure, for increasing forcing frequency, symmetric period- $(1,1,1)$ attractors are observed for $\omega \in(0.01000,0.19966)$ and typical trajectories are shown in Fig. 9(a) for $\omega=0.03616$, in Fig. 9(b) for $\omega=0.11246$ and in Fig. 9(c) for $\omega=0.16260$. At $\omega=0.19966$, a grazing event (GR) occurs and the response turns into a symmetric period- $(1,2,2)$ orbit. Shortly after, another grazing event (GR) takes place at $\omega=0.21274$, turning the response back into a symmetric period-(1,1,1) orbit. A typical trajectory is shown in Fig. 9(d) for $\omega=0.40022$. This attractor is observed up to $\omega=0.50279$, where a saddle-node bifurcation (SN) occurs. At this value, the system jumps to non-impacting period- $(1,0,0)$ attractor observed to the end of the parameter window. A typical trajectory is shown in Fig. $9(\mathrm{~g})$ for $\omega=0.99972$. 


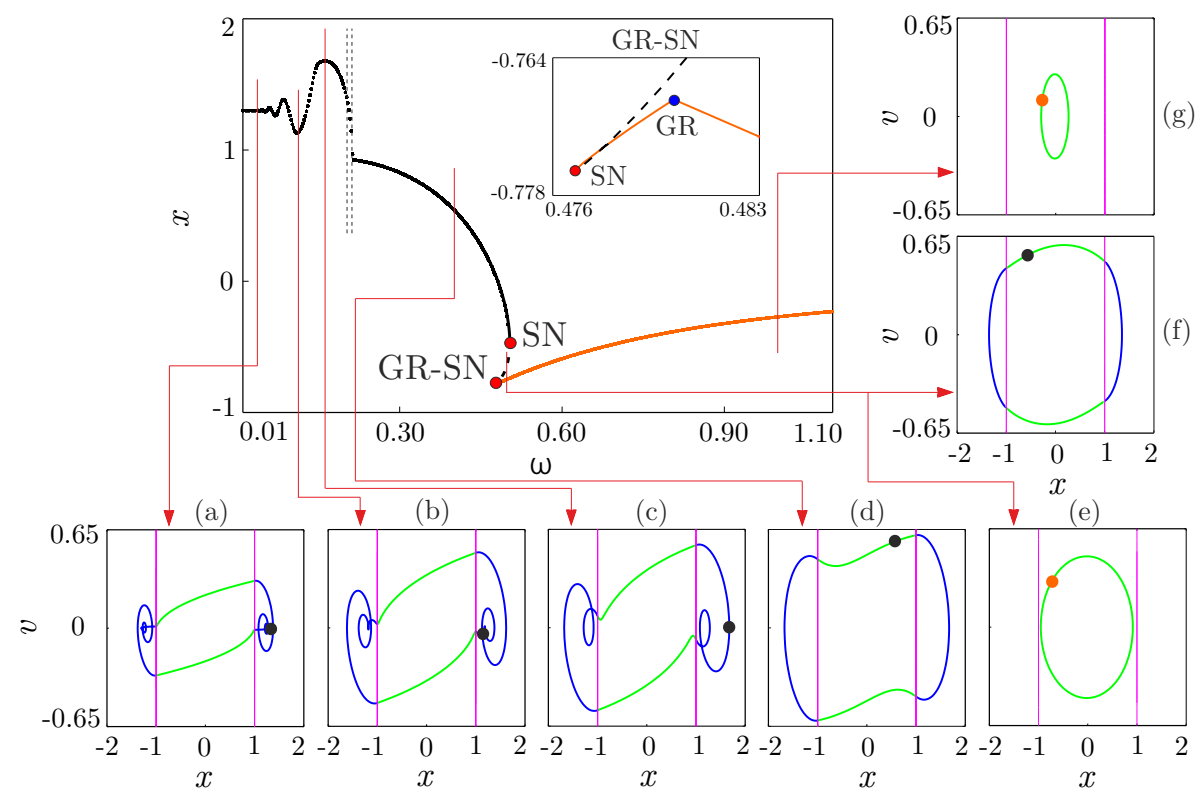

Figure 9: Bifurcation diagram of displacement as a function of the forcing frequency, $\omega$, computed for $a=0.3, e=1$ and $\xi=0.2$, where the control parameter was increased and decreased. To locate the unstable branch, numerical continuation was employed. Co-existing attractors are in black and orange, and stable and unstable periodic orbits are presented by solid and dashed lines respectively. Labels SN denote saddle-node bifurcation points. Grazing events are represented by the label GR and by dashed vertical lines. Additional panels show trajectories and Poincaré maps on the $(x, v)$ planes computed for the following frequencies: (a) 0.03616, (b) 0.11246, (c) 0.16260, (d) 0.40022, (e)0.50050, (f) 0.50050 and (g) 0.99972. There, operation in No-Contact mode is in green, whereas in Contact-Up and Contact-Down is in blue; points of the Poincaré maps are in the same colours used in the bifurcation diagram; and magenta vertical lines denote the discontinuity boundaries.

As decreasing forcing frequency is considered, a non-impacting period- $(1,0,0)$ attractor (typical trajectory is shown in Fig. $9(\mathrm{~g})$ for $\omega=0.99972)$ is observed up to $\omega=0.48013$, where the orbit comes into grazing contact (GR) with both boundaries. This grazing event is visible in the zoom-up window placed in the top right-hand side of the bifurcation diagram. Following this event, impacting period-(1,1,1) attractor is observed in the interval $\omega \in(0.48009,0.48013)$. At $\omega=0.48009$, another saddle-node (SN) bifurcation occurs, where the response jumps to the period-(1,1,1) response described earlier.

Computed by numerical continuation, the branch of unstable symmetric period- $(1,1,1)$ responses, is obtained in the interval $\omega \in(0.48009,0.50279)$. This branch connects the presented saddle-node points. Fig. 9 (f) demonstrates a typical trajectory of the unstable impacting period- $(1,1,1)$ response for $\omega=0.50050$ whereas Fig. 9 (e) shows the trajectory of stable co-existing non-impacting period-(1,0,0) response at the same forcing frequency.

The results presented in Figs 8 and 9 demonstrate that in the considered frequency range for the small value of damping ratio $\xi=0.02$, the system response varies from chaotic to various types of periodic orbits, whereas for a larger value $\xi=0.20$, the motion always settles to a periodic response. To determine the value of the damping ratio at which the chaotic motion is no longer present in the 
considered frequency interval, $\omega=(0.01,1.10)$, a number of additional simulations was undertaken. As a result, it was obtained that chaotic motion is observed in the dynamics of the system for values of $\xi$ between 0.020 and 0.139 .

To gain more insight into the system behaviour at lower frequencies for the small value of damping ratio $\xi=0.02$, the region of $\omega \in(0.124,0.140)$ (a subset of the bifurcation diagram shown in Fig. 8) was considered and it is presented in Fig. 10.

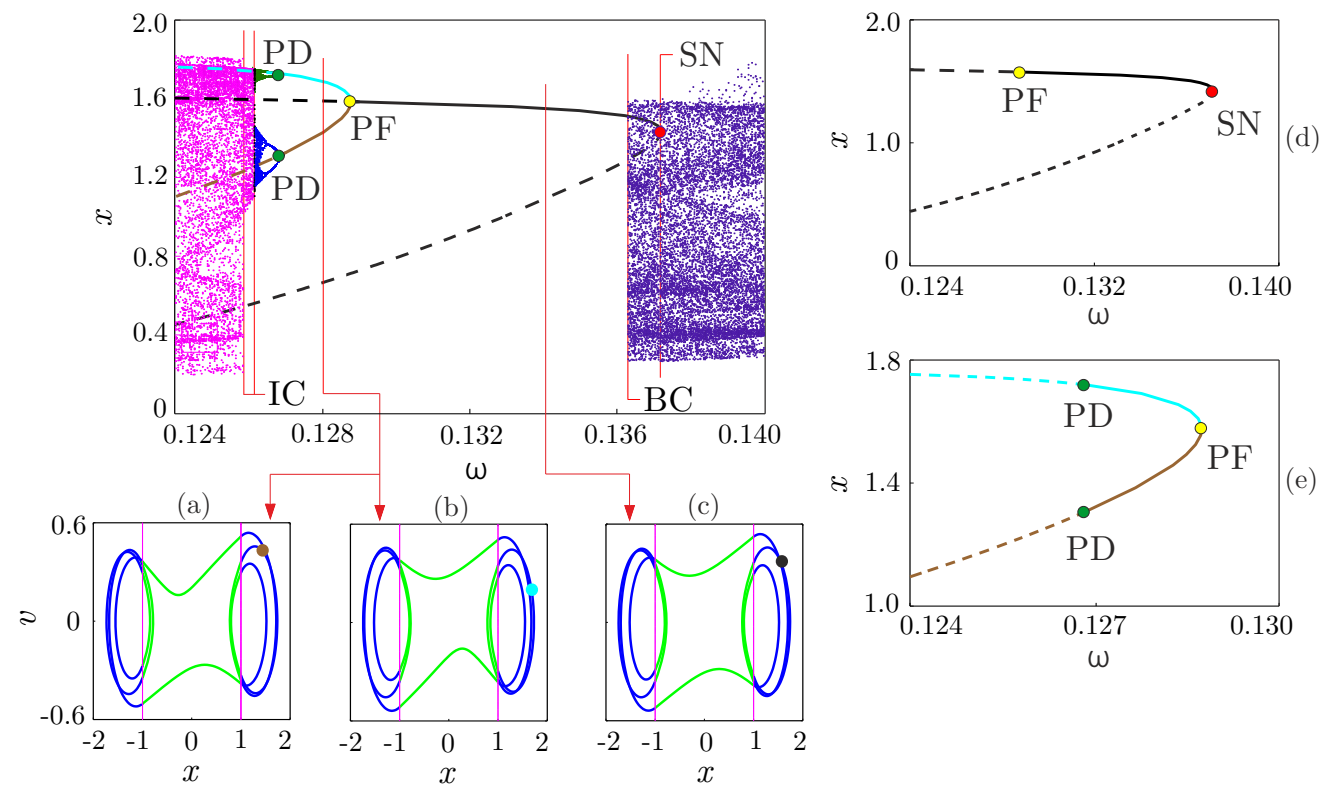

Figure 10: Bifurcation diagram of displacement as a function of the forcing frequency, $\omega$, (zoom-up of Fig. 8) computed for $a=0.3, e=1$ and $\xi=0.02$, where the control parameter was increased and decreased. To locate unstable branches, numerical continuation was employed. Attractors are in magenta, blue, dark green, brown, cyan, black and purple colours, and stable and unstable periodic orbits are presented by solid and dashed lines respectively. Labels SN, PD and PF denote saddle-node, period-doubling and pitchfork bifurcation points. Labels IC and BC mark the onset of interior crisis and boundary crisis respectively. Additional panels show trajectories and Poincaré maps on the $(x, v)$ planes computed for the following frequencies: (a) 0.12800 , (b) 0.12800 and (c) 0.13402 . There, operation in No-Contact mode is in green, whereas in Contact-Up and Contact-Down is in blue; points of the Poincaré maps are in the same colours used in the bifurcation diagram; and magenta vertical lines denote the discontinuity boundaries. Panels (d) and (e) demonstrate co-existing orbits obtained by numerical continuation.

As can be seen from this figure, in this frequency range the system exhibits both periodic and chaotic behaviour and there are a number of co-existing attractors. Typical trajectories of the recorded periodic responses are shown in Fig. 10(a) and 10(b) for two co-existing asymmetric period-(1,3,3) orbits and in Fig. 10(c) for the symmetric period-(1,3,3) orbit. The latter exists between saddle-node (SN) bifurcation point at $\omega=0.13715$ and pitchfork $(\mathrm{PF})$ bifurcation recorded at $\omega=0.12874$ as shown in Fig. 10(d). The co-existing period-(1,3,3) attractors undergo period-doubling bifurcation at $\omega=0.12682$ as demonstrated in Fig. 10(e) where stable and unstable orbits are shown. To unveil the unstable branches, numerical continuation was performed using the signature $\left\{I_{4}, I_{1}, I_{4}, I_{2}, I_{6}, I_{2}, I_{6}, I_{2}, I_{6}, I_{1}, I_{4}, I_{1}, I_{5}\right\}$ (the 
definition of the segments $I_{n}$ is given in Table 2). Interior crisis (IC) bifurcations at the frequencies values $\omega=0.12582$ and 0.12614 , and boundary crisis (BC) bifurcation at $\omega=0.13632$ are also observed.

The evolution of the basins of attraction presented in Fig. 11 (computed by ABESPOL) allows for a better understanding of the bifurcation structure depicted in Fig. 10. Below, the co-existing attractors obtained at $\omega=0.13700$ are considered and followed as the frequency increases and then decreases to explain the observed changes on the system reponse. The basins of attraction for these co-existing period$(1,3,3)$ and chaotic responses are presented in Fig. 11(g) where a chaotic attractor is shown in purple with its basin in turquoise and the period- $(1,3,3)$ attractor is in black with its basin is in red. Increases in the value of $\omega$ lead the system to a saddle-node bifurcation of the period- $(1,3,3)$ attractor at $\omega=0.13715$, beyond which this periodic attractor no longer exists as shown in Fig. 11(h).

(a)

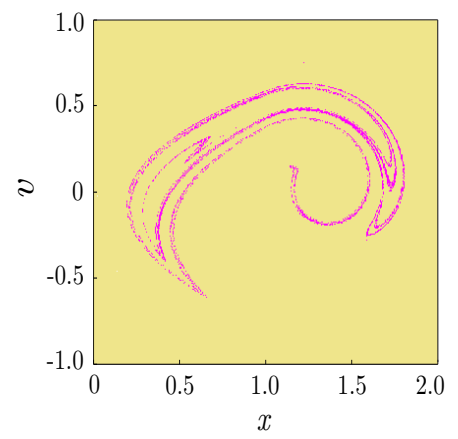

(e)

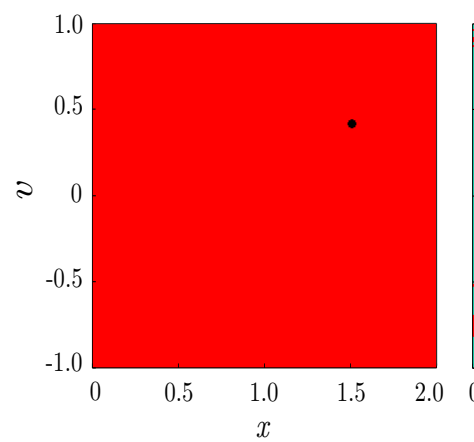

(b)

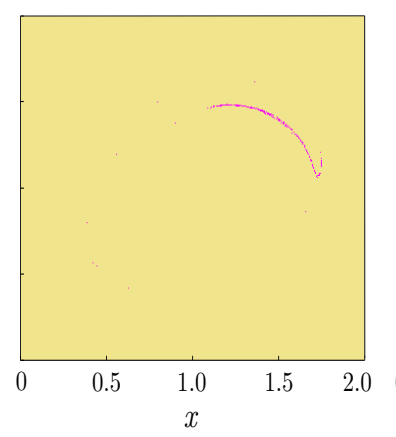

(f)

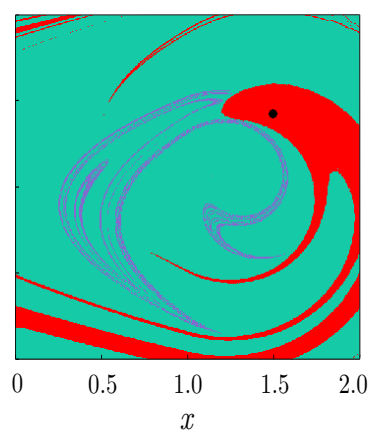

(c)

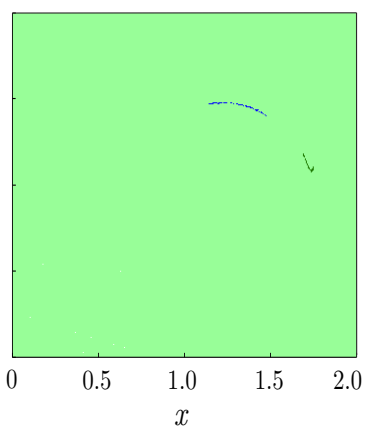

(g)

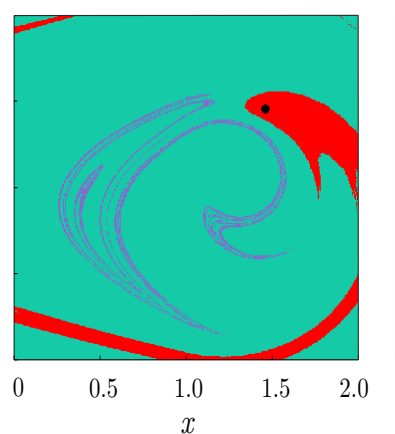

(d)

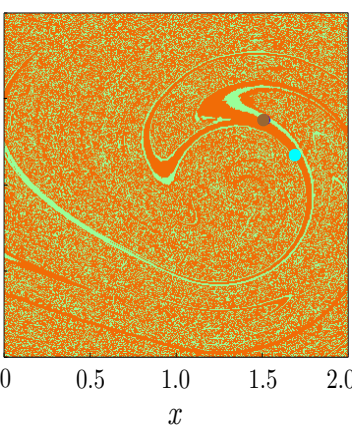

(h)

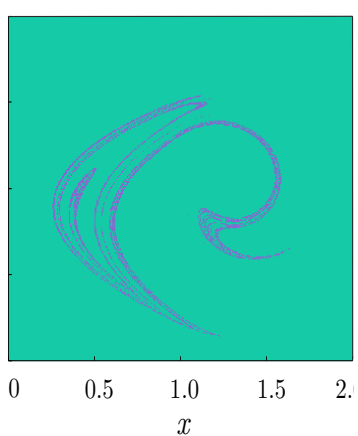

Figure 11: Basins of attraction in the frequency range of $\omega \in(0.124,0.140)$ presented in Fig.10, computed at the frequencies (a) 0.12560 , (b) 0.12610 , (c) 0.12624, (d) 0.12800 , (e) 0.13627, (f) 0.13640, (g) 0.13700 and (h) 0.13720 . Attractors are displayed in the same colours used in the bifurcation diagram. In (a) and (b), the chaotic attractor in magenta has its basin in khaki. In (c), the chaotic attractor in blue has its basin in light green while the chaotic attractor in dark green has its basin sufficiently small to not be visible on the picture. The period-1 attractors coloured brown and cyan in (d) have their basins in light green and orange respectively. The period-1 attractor painted black in (e), (f) and (g) has its basin in red. The chaotic attractor coloured purple in (f), (g) and (h) has its basin in turquoise.

As the frequency decreases from $\omega=0.13700$, the chaotic attractor in purple approaches the saddle point in the basin boundary while the basin in red expands in the shape of needles towards the chaotic attractor as seen in Fig. 11(f). When the unstable period-1 orbit and the chaotic attractor collide at 
$\omega=0.13632$, a boundary crisis occurs, leading the chaotic attractor and its basin to vanish as shown in Fig. 11(e), where the period-(1,3,3) attractor appears alone. As the frequency $\omega$ continues to decrease, a pitchfork bifurcation takes place at $\omega=0.12874$, leading to appearance of two co-existing asymmetric period-(1,3,3) attractors as shown in Fig. 11(d). Here, the attractor in brown has its basin in light green and the attractor in cyan has its basin in orange. These two co-existing attractors (shown in Fig. 10(a) and 10(b)) undergo simultaneous period-doubling bifurcations at $\omega=0.12682$, which are followed by the co-existence of two chaotic attractors as shown in Fig. 11(c). In Fig. 11(c), the basin of the chaotic attractor in blue is in light green, and the basin of the attractor in dark green is sufficiently small to not be visible. Then, the coexisting chaotic attractors from Fig. 11(c) give rise to the chaotic attractor in magenta shown in Fig. 11(b) whose basin is in khaki. This occurs via an interior crisis bifurcation at $\omega=0.12614$. Finally, another interior crisis takes place at $\omega=0.12582$ leading to the enlargement of the chaotic attractor as seen in Fig. 11(a).

\section{Closing remarks and future work}

This paper presents an overview of the nonlinear dynamic responses of a harmonically excited linear oscillator with a play, which is modelled as a single-degree-of-freedom system and undergoing non-smooth events. The non-smoothnesses come from intermittent contacts between the mass and the spring when a gap is exceeded. Three operational modes were defined as well as the discontinuity boundaries where the non-smoothnesses take place. Then, all possible events of the system were in turn defined in order to analyse the system with our newly developed in-house Matlab-based computational suite ABESPOL [1]. As well as trajectories and Poincaré sections, bifurcation diagrams and basins of attraction were computed for sets of parameter values displaying a number of distinct responses. Each of the system parameter was considered as a control parameter except the gap. To unveil unstable reponses along with detection and location of bifurcation points, ABESPOL routines were used together with the continuation core $\mathrm{COCO}[30]$.

The computed responses included both periodic and chaotic orbits and classical bifurcations, such as saddle-node, pitchfork and period-doubling, were detected. In addition, a detailed analysis of nonimpacting and impacting orbits was carried out to investigate grazing incidences. The case of grazing incidence where an impacting orbit undergoes an increase or a decrease in the number of impacts with the boundaries were studied as well.

An attention was also given to the occurrence of crisis phenomena, that is, boundary and interior crisis bifurcations. As defined in the literature, it was observed that the boundary crisis occurred when 
a chaotic attractor touched an unstable periodic orbit, with which the chaotic attractor and its domain of attraction vanished and became part of the basin of the co-existing stable periodic response. With respect to the interior crisis, it is known that such a bifurcation gives rise to an enlargement of a chaotic attractor as a result of the collision between this attractor and an unstable one. However, in one of our computed bifurcation diagrams, we found a parameter region for which such a enlargement of a chaotic attractor occurred without a collision between the unstable orbit and the chaotic attractor.

As for future work, the interior crisis where the collision between the unstable orbit and the chaotic attractor did not occur will be investigated. In addition, more in-depth analysis of grazing events will be undertaken both numerically and analytically to get more insight into grazing-induced bifurcations for this system.

\section{Acknowledgements}

This work was supported by the National Secretariat of Science, Technology and Innovation of Ecuador (SENESCYT); the Escuela Superior Politécnica del Litoral of Ecuador (ESPOL); the National Natural Science Foundation of China $(11272268,11572263)$ and Scholarship of China. A.S.E. Chong and Y. Yue acknowledge the hospitality of the Centre of Applied Dynamics Research at the University of Aberdeen.

\section{References}

[1] A.S.E. Chong. Numerical modelling and Stability Analysis of non-smooth dynamical systems. PhD thesis, University of Aberdeen, 2016.

[2] S.W. Shaw and P.J. Holmes. A periodically forced piecewise linear oscillator. Journal of Sound and Vibration, 90(1):129-155, 1983.

[3] G.X. Li, R.H. Rand, and F.C. Moon. Bifurcations and chaos in a forced zero-stiffness impact oscillator. International Journal of Non-linear Mechanics, 25(4):417-432, 1990.

[4] M. Kleczka, E. Kreuzer, and C. Wilmers. Crisis in mechanical systems. In Proc. Int. Conf. Nonlinear Dynamics in Engineering, pages 141-148. Springer, 1990.

[5] C. Grebogi, E. Ott, and J. Yorke. Crises, sudden changes in chaotic attractors, and transient chaos. Physica D, 7:181-200, 1983.

[6] Y. Ueda. Explosion of strange attractors exhibited by Duffing's equation. Annals of the New York Academy of Sciences, 357:422-434, 1980. 
[7] A.C.J. Luo and S. Menon. Global chaos in a periodically forced, linear system with a dead-zone restoring force. Chaos, Solitons and Fractals, 19:1189-1199, 2004.

[8] M. Wiercigroch. Bifurcation analysis of harmonically excited linear oscillator with clearance. Chaos, Solitons and Fractals, 4(2):297-303, 1994.

[9] A. Kaharaman and R. Singh. Nonlinear dynamics of a spur gear pair. Journal Sound and Vibration, 142(1):49-75, 1990.

[10] S. Theodossiades and S. Natsiavas. Non-linear dynamics of gear-pair systems with periodic stiffness and backlash. Journal of Sound and Vibration, 229(2):287-310, 2000.

[11] S.L.T. de Souza, I.L. Caldas, R.L. Viana, and J.M. Balthazar. Sudden changes in chaotic attractors and transient basins in a model for rattling in gear boxes. Chaos, Solitons and Fractals, 21:763-772, 2004.

[12] S. Natsiavas. Periodic response and stability of oscillators with simetric trilinear restoring force. Journal of Sound and Vibration, 134(2):315-331, 1989.

[13] A.B. Nordmark. Non-periodic motion caused by grazing incidence in an impact oscillator. Journal of Sound and Vibration, 145(2):279 - 297, 1991.

[14] H. Dankowicz and A.B. Nordmark. On the origin and bifurcations of stick-slip oscillations. Physica D: Nonlinear Phenomena, 136(3-4):280-302, 2000.

[15] J. Molenaar, J.G. De Weger, and W. Van De Water. Mappings of grazing-impact oscillators. Nonlinearity, 14(2):301-321, 2001.

[16] H. Dankowicz and X. Zhao. Local analysis of co-dimension-one and co-dimension-two grazing bifurcations in impact microactuators. Physica D, 202:238-257, 2005.

[17] M. di Bernardo, M.I. Feigin, S.J Hogan, and M.E. Homer. Local analysis of c-bifurcations in ndimensional piecewise smooth dynamical systems. Chaos, Solitons and Fractals, 10(11):1881-1908, 1999.

[18] H. Nusse, E. Ott, and J. Yorke. Border collision bifurcations: An explanation for observed bifurcation phenomena. Physical Review E, 49:1073-1076, 1994.

[19] S. Banerjee and C. Grebogi. Border collision bifurcations in two-dimensional piecewise smooth maps. Physical Review E, 59:4052-4061, 1999. 
[20] M. di Bernardo, C.J. Budd, and A.R. Champneys. Normal form maps for grazing bifurcations in n-dimensional piecewise-smooth dynamical systems. Physica D: Nonlinear Phenomena, 160(34):222-254, 2001.

[21] M. di Bernardo, P. Kowalczyk, and A.B. Nordmark. Bifurcations of dynamical systems with sliding: Derivation of normal-form mappings. Physica D: Nonlinear Phenomena, 170(3-4):175-205, 2002.

[22] Y. Ma, M. Agarwal, and S. Banerjee. Border collision bifurcations in a soft impact system. Physical Letter A, 354:281-287, 2006.

[23] Y. Ma, J. Ing, S. Banerjee, M. Wiercigroch, and E. Pavlovskaia. The nature of the normal form map for soft impacting systems. International journal of Nonlinear Mechanics, 43:504-513, 2008.

[24] A. Stensson and A.B. Nordmark. Experimental investigation of some consequences of low velocity impacts in the chaotic dynamics of a mechanical system. Philosophical transactions of Royal Society London A, 347:439-448, 1994.

[25] T.P. Piiroinen, L.N. Virgin, and A.R. Champneys. Chaos and Period-Adding: Experimental and Numerical Verification of the Grazing Bifurcation. Journal of Nonlinear science, 14:383-404, 2004.

[26] J. Ing, E. Pavlovskaia, and M. Wiercigroch. Dynamics of a nearly symmetrical piecewise linear oscillator close to grazing incidence: modelling and experimental verification. Nonlinear Dynamics, 46:225-238, 2006.

[27] J. Ing, E. Pavlovskaia, M. Wiercigroch, and S. Banerjee. Experimental study of impact oscillator with one-sided elastic constraint. Philosophical Transactions of the Royal Society A: Mathematical, Physical and Engineering Sciences, 366(1866):679-704, 2008.

[28] S. Banerjee, J. Ing, E. Pavlovskaia, M. Wiercigroch, and R.K. Reddy. Invisible grazings and dangerous bifurcations in impacting systems: The problem of narrow-band chaos. Physical Review E, 79(037201), 2009 .

[29] E. Pavlovskaia, J. Ing, M. Wiercigroch, and S. Banerjee. Complex dynamics of bilinear oscillator close to grazing. International Journal of Bifurcation and Chaos, 20(11):3801-3817, 2010.

[30] H. Dankowicz and F. Schilder. Recipes for Continuation. SIAM, 2013.

[31] M. Wiercigroch. Modelling of dynamical systems with motion dependent discontinuities. Chaos, Solitons and Fractals, 11(13):2429-2442, 2000. 
[32] H. Dankowicz and P. Thota. TC-HAT: A novel toolbox for the continuation of periodic trajectories in hybrid dynamical systems. SIAM Journal on Applied Dynamical Systems, 7(4):283-322, 2008. 